\title{
Enhancing detection of nitroaromatic vapors by utilizing polymer coatings on quartz crystal microbalances having strong dipoles
}

\author{
Timothy Ponrathnam ${ }^{1,3}$, Junghwan $\mathrm{Cho}^{2}$, Pradeep U. Kurup ${ }^{2}$, Jayant $\mathrm{Kumar}^{3,4}$ and \\ Ramaswamy Nagarajan ${ }^{1,3^{*}}$ \\ ${ }^{1}$ Department of Plastics Engineering, University of Massachusetts Lowell, Lowell, MA 01854 \\ ${ }^{2}$ Department of Civil and Environmental Engineering, University of Massachusetts Lowell, Lowell, MA 01854 \\ ${ }^{3}$ Center for Advanced Materials, University of Massachusetts Lowell, Lowell, MA 01854 \\ ${ }^{4}$ Department of Physics and Applied Physics, University of Massachusetts Lowell, Lowell, MA 01854 \\ E-mail:Ramaswamy_Nagarajan@uml.edu
}

\begin{abstract}
A novel class of PDMS based polymers containing azobenzene side chains were synthesized and evaluated as coatings for quartz crystal microbalance (QCM) based sensors to detect nitroaromatic vapors. The polymers contain functional groups (p-substituted azobenzene moieties and hydroxyl groups) that can interact with compounds with large dipole moments such as nitroaromatic chemicals through a combination of permanent dipole-dipole interactions and hydrogen bonding. A QCM sensor array was prepared by coating different polymers onto the surface of a quartz crystal and evaluating the response of the each coating to saturated vapors of a variety of analytes such as toluene, octane, 2-nitrotoluene, nitrobenzene, and 2,4 dinitrotoluene. The incorporation of azobenzene moieties in the polymer structure increased the sensor response towards the nitroaromatic analytes. Pattern recognition techniques such as principal component analysis (PCA) and decision tree learning methods were applied to evaluate the sensor response and predict analyte identity. A classification accuracy of $96 \%$ was obtained using classification and regression trees (CART)

2015-03-13

Revised Draft
\end{abstract}

(C) 2015. This manuscript version is made available under the Elsevier user license 
based learning. This novel approach opens new possibilities in ultrasensitive detection of chemical vapors with significant dipole moments.

\section{Keywords}

QCM sensors, Vapor sensing, Nitroaromatics, DNT, Explosives, Explosive precursor, Taggants, Decision trees

\section{Introduction:}

Common nitroaromatic explosives have low vapor pressures at room temperature and therefore detection of trace quantities of these compounds under ambient conditions requires extremely sensitive detection technologies [1]. Sensors based on ion-mobility spectrometry (IMS) are often used for the detection of explosives at airport security check points [2]. IMS based devices have excellent sensitivity that allows the detection of trace quantities of these explosives. However, as with other detection techniques IMS also can exhibit false positive responses [3].

IMS has been used to determine the charge to mass ratio and mobility ratios of over 22 compounds of interest to national security (including explosives, illicit drugs, and chemical warfare agents) [4]. This study indicated that chemicals present in cosmetic products (including fragrances, cosmetics, sunscreen lotions) commonly encountered in airports could potentially interfere with detection analytes of interest (explosives, drugs, chemical warfare agents). More importantly 17 suspected contaminants found in airport settings decreased the sensitivity of TNT detection [5]. Thus, there exists the need for alternate sensing technology platforms that can be integrated with systems that are portable and low-cost to provide improved sensitivity and specificity for the detection of nitroaromatics. 
The success of canines in chemical identification has motivated research in 'electronic nose' systems that attempt to mimic the canine olfactory system. Electronic nose systems usually comprise several sensing elements, each of which can detect different chemicals. The combined signals obtained from these sensors are analyzed using intelligent signal processing algorithms and complex pattern recognition tools to substantially minimize false positive responses while preserving the sensitivity of detection [6].

Arrays of fluorescent polymer sensors [7-9], metal oxide sensors [10,11], quartz crystal microbalance (QCM) and surface acoustic wave (SAW) based piezoelectric mass sensors [12] have been investigated over the past decade as possible alternatives to the already commercially available ion-mobility based sensors. QCM based sensors have been reported for the vapor phase detection of chemical warfare agents [13-16]. These devices have demonstrated sensitivity to various organic chemical vapors. QCM sensors are compact and are capable of being miniaturized for the on-site detection of nitro aromatic explosives and explosive precursors.

The sensitivity and selectivity of the piezoelectric mass sensors (QCM and SAW) can be enhanced by the incorporation of surface coatings [17]. The surface coating (polymers or metal oxides) that form the active layer can interact with test analytes through various mechanisms that include solvation, secondary interactions such as permanent dipole-dipole interactions and hydrogen bonding.

Most polymer coatings designed to serve as the active layer for these sensors reported to date utilize hydrogen bonding interactions [17] to adsorb chemical analytes. Polymer coatings containing hydrogen-bond acidic moieties have been used for the detection of organophosphorus nerve gas simulants such dimethyl methylphosphonate (DMMP) [18,19]. 
Incorporation of fluorinated alcohols or fluorinated phenols (groups that exhibit strong hydrogen bond acidity) in polymers has been demonstrated to be beneficial for obtaining sensitive coatings capable of detecting chemical vapors through hydrogen bonding [20] interactions. Hexafluoroisopropanol functionalized siloxane based polymers have been synthesized and evaluated as vapor sensitive coatings for use with chemical sensors. While these strong hydrogen-bond acidic polymers have demonstrated strong affinity for nerve gas simulants, they are not very sensitive for the detection of nitroaromatics such as DNT [20].

Nitroaromatic analytes like nitrobenzene, 2-nitrotoluene and 2,4 dinitrotoluene (DNT) have large permanent dipole moments (in the range of $3-6$ Debye) as shown in figure 1 , and can interact with functional coatings through dipole-dipole and dipole-induced dipole interactions.

One of the strategies we have reported for improving the sensitivity in detection of analytes with high dipole moment is to utilize polymer coatings containing functional group that exhibit large dipole moments. In this regard, p-nitroazobenzene moieties have an electric dipole of about 4.45 D [21], while the p-carboxylic acid azobenzene moieties have an electric dipole moment of 2.9 D [22]. Thus nitroaromatic analytes could interact with the azobenzene functional groups via dipole-dipole interactions.

We have previously reported that a polymeric coating containing functional groups with strong dipole moments can indeed interact with explosive vapors. Polypropylene glycol based coatings containing p-nitroazobenzene moieties were synthesized and tested as active layers in QCM sensors for the detection of nitroaromatics [23].

The p-nitroazobenzene functionalized PPG coating (see figure 2) exhibited significantly higher response to saturated nitroaromatic vapors when compared to the unfunctionalized PPG coating polymer without the azobenzene moieties (see figure 3). Polymer coatings used 
as active layers for QCM and SAW sensors must have glass transition temperatures below the operating temperature of the sensing device [17]. While the PPG containing polymers exhibited some response to nitroaromatics, there were several shortcomings in terms of reversibility of the sensor response. The polymer backbone was not as porous as desired to maximize the interactions of the azobenzene moieties with analytes of interest. PDMS offers better porosity and flexibility to improve dipole-analyte interactions, therefore improving sensitivity of detection. Polysiloxane based materials modified with aniline moieties have been evaluated as functional modifications for QCM sensors used for the detection of nitroaromatic vapors [24]. Nitroaromatic compounds such as nitrobenzene, dinitrobenzene, DNT, trinitrobenzene and trinitrotoluene were tested in this study. In these coatings, the underlying mechanism of interaction is attributed to H-bonding between the aniline groups present in the polymer and the nitro groups present on the analyte.

In order to test the hypothesis that a coating with lower glass transition temperature $\left(\mathrm{T}_{\mathrm{g}}\right)$ and better diffusivity can indeed help with interactions of analytes with organic moieties with high dipole moment, p-nitroazobenzene functionalized PDMS based polymer was synthesized and tested in a Surface Acoustic Wave (SAW sensor). Preliminary studies evaluating the coating response to nitroaromatic explosive and explosive precursors indicated better sensitivity [25].

In this study, poly(dimethyl siloxane) (PDMS) based polymer coatings containing a combination of moieties that can hydrogen bond with analytes (specifically nitroaromatics) and functional groups with large dipole moment (p-substituted azobenzene moieties) is reported. The polymers synthesized were coated on QCM sensors and the effectiveness of the QCM sensor array in detection of trace quantities of nitroaromatic analyte vapors (2- 
nitrotoluene, nitrobenzene and 2,4 DNT) as well other organic analytes such as toluene, and octane was evaluated. Decision tree based learning methods were applied to evaluate the performance of sensor array.

Decision tree-based learning methods are powerful and popular machine learning tools for classification and making numeric predictions [26]. The attractiveness of decision tree-based learning methods over other machine learning techniques such as neural networks, include their ease of use, and generation of easily inspected models with rules that can be readily understood. Models created using this method display internal nodes that form a tree-like structure (hence the name). The internal nodes (test nodes) represent tests on a feature's (variable's) values, and the leaf nodes (terminal or decision nodes) are assigned classes. Decision trees are learned by recursively selecting the variables that "best" sort the data into non-overlapping subsets. As the splitting continues, the variables chosen for splitting the data are arranged into a tree, with the first variable chosen for splitting forming the root of the tree, and the variables used to split the subsets formed by the values of the first variable becoming child nodes of the first variable, and so on. This recursive splitting continues until a subset's members have the same class or the subset's size is less than a user-supplied threshold. After training, the tree may be used for classification by traversing from the top node, called the root node, down to a leaf node, using the values of the case's variables to determine the path taken to reach that node. The class assigned to this leaf node would be the class predicted for the case. 


\section{Experimental}

\subsection{Reagents and Materials}

Diglycidyl ether terminated Poly (dimethyl siloxane) $\left(M_{n}=980\right)$, aniline, zinc chloride were purchased from Sigma-Aldrich (St. Louis, MO) and were used as received. Acetonitrile (ACS grade) and chloroform (ACS grade) were purchased from Pharmco-AAPER (Brookfield, CT) and were distilled prior to use. Sodium nitrite, p-nitroaniline, glacial acetic acid, sulfuric acid, sulfamic acid, N. N'-dimethylformamide and sodium bicarbonate were purchased from Sigma-Aldrich and were used as received. The test analytes 2-nitrotoluene (analytical grade), nitrobenzene (analytical grade) were purchased from Sigma-Aldrich (St. Louis, MO) and were used as received. 2,4 DNT (97\% pure) was purchased from Sigma-Aldrich (St. Louis MO) and was recrystallized from anhydrous ethanol prior to use.

\subsection{Characterization}

FTIR spectroscopy was carried out on a Thermo Scientific Nicolet 4700 with a Smart Orbit ATR accessory. Molecular weight was estimated using an Agilent Gel Permeation Chromatography (GPC) instrument with a RI detector calibrated with poly(dimethyl siloxane standards). GPC analysis was performed using a pair of Phenomenex Phenogel columns (Column a pore size 1000 angstrom, column b pore size 100 angstrom) connected in tandem.

Chloroform was used as the mobile phase at a flow rate of $1 \mathrm{ml} / \mathrm{min}$. The ${ }^{1} \mathrm{H} \mathrm{NMR}$ spectra were collected using a Bruker $500 \mathrm{MHz}$ NMR spectrometer. The solvent used was deuterated methanol. (Cambridge Isotope Labs Inc.)

2015-03-13

Revised Draft 


\subsection{Synthesis of PDMS-AN polymer}

The synthesis of the precursor polymer (PDMS-AN) was carried out according to the scheme shown in figure 4. Equimolar quantities of poly(dimethyl siloxane) (1.96 g, $2 \mathrm{mmol}$ ) and aniline $(0.186 \mathrm{~g}, 2 \mathrm{mmol})$ were dissolved in $30 \mathrm{ml}$ of acetonitrile. Catalytic amounts of zinc chloride $(5 \mathrm{~mol} \%)$ were added to the reaction mixture to catalyze the reaction. The reactants were stirred and heated to $85{ }^{\circ} \mathrm{C}$ for 48 hours. The reaction mixture was then cooled to room temperature. The reaction mixture was then concentrated by distilling the solvent under reduced pressure to remove the acetonitrile. This was followed by dissolving the reaction mixture in glacial acetic acid. The polymer solution was subsequently dialyzed using a $(2,000 \mathrm{Mw})$ cut off dialysis bag to remove lower molecular impurities in the system. Upon completion of the dialysis, the polymer solution was once again concentrated by distillation of solvent at reduced pressure. The polymer was washed with water several times, followed by extraction with chloroform. The weight average molecular weight of the synthesized poly(dimethyl siloxane) polymer was estimated as 20,250 with a polydisperity index of 1.8 when compared with poly(dimethyl siloxane) standards.

Spectroscopic Characterization: Fourier Transform Infrared Spectroscopy (ATR) v $\left(\mathrm{cm}^{-1}\right)$ 2800, 1590, 1510, 1265,1010, 760, 660.

${ }^{1} \mathrm{H}$ NMR (500 MHz, Acetone-d6) 7.16(d, 2H), 6.84(dd, 2H), 6.62(d, 1H), 4.07(d, 2H), 3.94(d, $1 \mathrm{H}), 3.65(\mathrm{~d}, 1 \mathrm{H}), 3.55-3.38(\mathrm{~m}, 10 \mathrm{H}), 1.74-1.64(\mathrm{~m}, 4 \mathrm{H}), 0.70-0.61(\mathrm{~m}, 4 \mathrm{H}), 0.13(\mathrm{~d}, 86 \mathrm{H})$

\subsection{Synthesis of PDMS-AN-NT polymer}

The synthesis of the polymer (PDMS-AN-NT) was carried out according to the scheme shown in figure 5. A diazonium salt of p-nitroaniline was prepared by adding an aqueous 
solution of sodium nitrite $(0.02 \mathrm{~g}, 0.289 \mathrm{mmol}$ in $1 \mathrm{ml}$ of water $)$ drop wise to a dispersion of pnitroaniline $(0.035 \mathrm{~g}, 0.25 \mathrm{mmol})$ in a homogeneous mixture of sulfuric acid $(0.030 \mathrm{ml})$ and glacial acetic acid $(0.5 \mathrm{ml})$. The mixture was stirred at $2{ }^{\circ} \mathrm{C}$ for $5 \mathrm{~min}$ before the excess nitrous acid was decomposed by addition of sulfamic acid $(0.025 \mathrm{~g}, 5 \mathrm{mmol})$ in a suitable amount of water. The diazonium salt solution was added drop wise into a solution of PDMSAN (0.214 g, 0.2mmol) dissolved in a chloroform- dimethylformamide mixture (3:2) at $2{ }^{\circ} \mathrm{C}$. After the solution was stirred at $2{ }^{\circ} \mathrm{C}$ for $12 \mathrm{~h}$, the mixture was poured into water under agitation while adding a few drops of hydrochloric acid. The polymer was further purified by extraction with chloroform several times and dried under vacuum for at least $24 \mathrm{~h}$.

Spectroscopic Characterization: Fourier Transform Infrared Spectroscopy (ATR) v (cm ${ }^{-}$ 1) $2800,1590,1510,1430,1265,1010,760,660$

\subsection{Synthesis of PDMS-AN-CA polymer}

The synthesis of the precursor polymer (PDMS-AN-CA) was carried out according to the scheme shown in figure 6. A diazonium salt of p-aminobenzoic acid was prepared by adding an aqueous solution of sodium nitrite $(0.02 \mathrm{~g}, 0.289 \mathrm{mmol}$ in $1 \mathrm{ml}$ of water $)$ drop wise to a dispersion of p-aminobenzoic acid $(0.035 \mathrm{~g}, 0.25 \mathrm{mmol})$ in homogeneous mixture of sulfuric acid $(0.030 \mathrm{ml})$ and glacial acetic acid $(0.5 \mathrm{ml})$. The mixture was stirred at $2{ }^{\circ} \mathrm{C}$ for 5 min before the excess nitrous acid was decomposed by addition of sulfamic acid $(0.025 \mathrm{~g}, 5 \mathrm{mmol})$ in a suitable amount of water. The diazonium salt solution was added drop wise into a solution of PDMS-AN (0.214 g, 0.2mmol) dissolved in a chloroform-dimethylformamide mixture $(3: 2)$ at $2{ }^{\circ} \mathrm{C}$. After the solution was stirred at $2{ }^{\circ} \mathrm{C}$ for $12 \mathrm{~h}$, the mixture was poured into water under agitation while adding a few drops of hydrochloric acid. The polymer was

2015-03-13

Revised Draft 
further purified by extraction with chloroform several times and dried under vacuum for at least $24 \mathrm{~h}$.

Spectroscopic Characterization: Fourier Transform Infrared Spectroscopy IR (ATR) v ( $\mathrm{cm}^{-}$

1) $2800,1750,1590,1510,1430,1265,1010,760,660$

\subsection{Experimental setup for the detection of analytes using the polymer coated QCM} sensor

The test setup used is shown in figure 7. AT-cut quartz crystals $(10 \mathrm{MHz})$ were purchased from International Crystal Manufacturing (Oklahoma City, OK). The frequency of oscillation was continuously monitored via a B\&K Precision (Yorba Linda, CA) model 1823A frequency counter. One side of the QCM crystal was coated with $5 \mu \mathrm{l}$ of a $100 \mu \mathrm{M}$ solution of polymer in chloroform via drop casting. The frequency change on coating the quartz crystals with the functional polymer coatings is shown in Table 1 .

The crystals were dried in a vacuum oven prior to use. The frequency counter was interfaced to the computer using a serial cable. A virtual interface written on LabVIEW was used for acquiring the data from the frequency counter over a period of time.

The QCM was suspended from the lid of an air-tight $20 \mathrm{ml}$ scintillation vial containing the analyte of interest as shown in figure 7 . The flask temperature was maintained at $30^{\circ} \mathrm{C}$ using an external oil bath. The baseline frequency response of the QCM was measured when an empty vial was fitted to the lid containing the QCM. For measuring the response of the polymer-coated QCM to saturated vapors of analyte the following procedure was followed. The analyte was first taken in a vial and allowed to equilibrate at $30^{\circ} \mathrm{C}$ for several hours prior to the test. This vial was fitted underneath the cap containing the QCM. In all cases, the 
frequency change was continuously monitored. To evaluate the efficiency of desorption of analytes from the polymer coated QCM (after exposure to the analyte), the sensor was allowed to equilibrate in ambient air while the frequency response was continuously monitored. This process was repeated several times for every analyte and the sensor response was observed as change in frequency $(\mathrm{Hz})$ upon exposure to the analyte for a specific duration (typically 5 minutes).

A typical response of a polymer coated QCM sensor (PDMS-AN-NT) to saturated vapors of an analyte (2-nitrotoluene) is shown below in figure 8 . The sensor was exposed for a total of five minutes to the analyte vapor. The sensor was then allowed to equilibrate in ambient air to allow the analyte to desorb from the surface of the sensor. By keeping the equilibration time and then subsequent analyte exposure time's constant, the repeatability of the sensor is evaluated.

As seen if figure 8 , the sensor performance is highly repeatable. The average sensor response to saturated vapors of $2 \mathrm{NT}$ is $2048 \mathrm{~Hz}$ with a standard error of $49.81 \mathrm{~Hz}$ or less than $2.5 \%$ of the sensor response.

\section{Results and Discussions:}

The interaction of analytes with the polymer coating present on the quartz crystal of the QCM leads to a mass increase ( $\Delta m$ ) which is manifested as a change in the frequency $\Delta f$ of

the crystal according to Sauerbrey's equation [27].

$$
\Delta f=-c \Delta m
$$


For a particular quartz crystal $\mathrm{C}$ is a constant that depends on the resonant frequency of the crystal, the area between the electrodes, the density of quartz, and the shear modulus of quartz for the AT cut crystal.

The density of interactions and strength of the interactions of the analyte vapor with the dipoles on the polymer coating depends on concentration of analyte and the dipole moments of the analyte and the functional groups on the polymer. At a given temperature, the concentration of the analyte in the gas phase is directly related to the vapor pressures.

The saturation vapor pressures (at $30{ }^{\circ} \mathrm{C}$ ), dipole moments and Hildebrand solubility parameters of the test analytes estimated using Hansen Solubility Parameters in Practice (HSPIP) software are listed in table 2. Octane is a saturated Hydrocarbon with a negligible net dipole moment and was used as one of the analytes because it is commonly present in many locations where sensing of explosives is necessary. The maximum frequency change observed on the surface of the different polymer coated QCM sensors on exposure to saturated vapors of the analyte are listed below in table 3 .

The saturated vapor pressure of toluene at $30^{\circ} \mathrm{C}$ is about $29000 \mathrm{ppm}$. A comparison of response of the three sensors to saturated vapors of toluene is shown in figure 9 below. Despite the large vapor pressure of toluene, the change in frequency on the QCM sensors is lower than expected (significantly lower than all analytes except DNT). This can be ascribed to the very low dipole moment of toluene $(0.36 \mathrm{D})$ due to which toluene has minimal dipoledipole interactions with the polymer coatings. On removal of the analyte, the sensor recovers back to the baseline frequency in less than five seconds. This reversibility is very important from a practical standpoint for reusability of the polymer coating in a sensing device. 
The overshoot (initial very large frequency change, followed by recovery to stabilize at a higher frequency) is characteristic of analytes such as toluene with very large vapor pressures. This behavior has also been confirmed by results published by other investigators in the past. [28]

Octane also has a higher saturation vapor pressure than the nitroaromatic analytes at $30^{\circ} \mathrm{C}$ (as shown in table 2). The comparison of the response of the three sensors to saturated vapors of octane is shown in figure 10 below. Unmodified PDMS is non-polar and has strong affinity to saturated hydrocarbons such as octane. The sensitivity of the precursor polymer coated sensor (PDMS-AN) to octane can thus be attributed to a solvent effect [29]. The PDMS-ANCA coated sensor showed the smallest frequency change in the presence octane vapors. This is attributed to the presence of the polar carboxylic acid moiety present in this coating, which lowers the sensitivity of the coating to non-polar hydrocarbon analytes such as octane.

All the nitroaromatic analytes selected in this study, have a reasonable permanent dipole moment that can be attributed to the presence of the nitro group in the molecule. As shown in table 3, as expected, sensors incorporating p-nitroazobenzene and p-carboxylic acid azobenzene moieties in the polymer structure, demonstrate an increased sensitivity to nitroaromatic analytes. The azobenzene moieties provide permanent dipole-dipole interactions to complement the sorption of analytes and polymer coating and hydrogen bonding interactions between the hydroxyl groups and the nitro groups of the nitroaromatic analytes. The comparison of the sensor response to saturated nitrobenzene is shown below in figure 11.

The response of the sensors to saturated vapors of 2-nitrotoluene is shown in figure 12 . On comparing the response of the three QCM sensors to nitrobenzene and 2-nitrotoluene it is observed that the overall frequency change of the sensors in presence of saturated vapor of 2- 
nitrotoluene was greater. However, saturated vapor pressures and dipole moment of nitrobenzene is greater than 2-nitrotoluene (see table 2). The greater affinity of the PDMS based polymer coatings to 2-nitrotoluene may be attributed to the greater affinity of the PDMS backbone to 2-nitrotoluene. (See table 2 showing smaller differences between the solubility parameters of different coatings and 2-nitrotoluene) This can be confirmed by comparing the response of the PDMS-AN coated sensor to both 2-nitrotoluene and nitrobenzene. In presence of 2-nitrotoluene vapors the PDMS-AN sensor demonstrated a larger frequency change of $1272 \mathrm{~Hz}$, while the frequency change in presence of nitrobenzene vapors was $1018 \mathrm{~Hz}$.

Among the QCM sensors utilized in this study, the PDMS-AN-NT coated sensors incorporating p-nitroazobenzene moieties demonstrated the maximum response in the presence of the nitroaromatic analytes. This can be ascribed to the higher dipole moment of the p-nitroazobenzene moiety present in the PDMS-AN-NT coated sensors (4.45D).

Among the nitroaromatic analytes studied in this investigation, $(2,4)$ DNT has the highest dipole moment (5.24 Debye). In comparison to nitrobenzene and 2-nitrotoluene, 2,4 DNT is a solid at the test temperature. The saturation vapor pressure of 2,4 DNT is significantly lower than the other analytes tested at $1.3 \mathrm{ppm}$ at $30^{\circ} \mathrm{C}$. The comparison of the sensor response to saturated vapors of 2,4 DNT is shown in figure 13 below.

As a result the sensor response to DNT is lower than the other nitroaromatic analytes. The precursor polymer PDMS-AN also showed fair sensitivity to 2,4 DNT. The nature of interaction between the nitroaromatic analytes and the precursor polymer coating could be based on hydrogen bonding between the hydroxyl groups in the polymer structure and the nitro groups in the analytes. [20] The recovery time (time taken for the frequency change of 
the QCM to revert back to the baseline frequency) is significantly larger in-case of the tests with DNT. This may be ascribed to several reasons as discussed below. Due to the lower vapor pressure of the 2,4 DNT, once the analyte is adsorbed onto the coating the time required for it to desorb from the sensor (at the end of testing in the absence of the analyte) into the environment will also be larger. This increase in the time required by the sensors to recover the baseline frequency is also larger in case of the coatings incorporating azobenzene moieties. This can be attributed to dipole-dipole interactions that are developed between the analyte and polymer coating in these sensors. The greatest response to saturated vapors of DNT was obtained using PDMS-AN-NT coated sensors incorporating the azobenzene moieties with a largest dipole moment.

The reproducibility of the sensor response was investigated by repeating the test five times. The comparison of the sensor response (frequency change) to analytes tested is summarized in figure 14 below. The sensor response is very reproducible as seen by the low magnitude of the standard deviations (error bars) figure.

In comparison to the poly(propylene glycol) based coatings evaluated in earlier studies [23] the poly(dimethyl siloxane) based coatings demonstrated faster response and recovery times. In addition the repeatability of the sensor response was better especially in case of 2,4 DNT.

Since, three different sensors (coatings on QCM sensor) were investigated in this study; a $25 \times 3$ (5 analytes $\times 5$ tests $\times 3$ coated $\mathrm{QCM}$ sensors) data matrix was prepared. In order to reduce the dimensionality of input space of the data matrix, principle component analysis (PCA) was performed to extract relevant key features. To obtain the PCA results, the covariance matrix was first calculated using the data matrix. Once the covariance matrix was determined, the Eigen vectors corresponding to the principal components were calculated by 
solving the characteristic equation of the covariance matrix. The PCA results are plotted in figure. 15 shows well separated for different chemical analytes (nitrobenzene, 2-nitrotoluene, toluene, DNT, octane). The five points located for each analyte group represents the five repetitions of the tests performed at the same concentration. The five groups of the data roughly characterize an area where the sensor response of the same analyte could possibly appear.

The presence of well separated clusters in the PCA demonstrates that by applying other pattern recognition techniques the sensor response can be used for prediction of analyte. The classification and regression tree (CART) algorithm was applied for the analyte prediction. Using the CART algorithm, the classification tree was trained and tested by 5-fold crossvalidation method with 25 data sets. The dataset was divided into 5 test folds so that each classification tree was trained using the remaining 20 measurements and then tested with the five test examples. Figure 16 shows a classification tree built by CART taking the inputs to be the frequency change of polymer coated QCM sensor array. A tree consisting of three internal nodes and five terminal nodes was generated and each terminal node indicates the analyte class.

Table 5 shows the confusion matrix obtained by the CART method. In this matrix, each column specifies the actual analytic sample being measured and each file indicates the number of times it was assigned to each type of nitroaromatic chemicals. For example, 2nitrotoluene (NT) vapor was classified correctly four times and misclassified as nitrobenzene (NB) vapor once. Since a 24 of the total 25 samples were identified correctly, the prediction tree model built by CART showed a classification result with an accuracy of $96 \%$. 


\section{Conclusions}

A novel class of PDMS-based polymer containing azobenzene side chains has been developed and tested for the detection of vapors. The polymers consist of functional groups (p-substituted azobenzene moieties and hydroxyl groups) that can interact with nitroaromatic compounds via a combination of permanent dipole-dipole interactions and hydrogen bonding. The polymers were coated on the surface of quartz crystal microbalance sensors that were used to detect saturated vapors of different chemical analytes. The sensor response to the analytes tested is highly reproducible. Typically an increase in the dipole moment and vapor pressure of an analyte caused greater frequency change (i.e. more adsorption of analyte on polymer) during testing. However, in case of nitrobenzene and 2-nitrotoluene the trend was reversed. This can be attributed to the greater affinity of the PDMS backbone to 2nitrotoluene. Principle component analysis of the sensor response showed well separated clusters. Using the sensor data, decision tree based learning techniques were utilized to accurately predict and classify the analyte. CART based learning techniques achieved a classification accuracy of $96 \%$ This sensing technique based on dipole-dipole interaction opens possibilities for the design of new types of functional coatings suitable for efficient detection of explosives and other precursor materials. Combining the response from these new types of sensors using pattern recognition techniques has been shown to significantly enhance the accuracy of identification of explosive vapors.

\section{Acknowledgements}

Financial support from the National Science Foundation (award number ECCS 0731125) is greatly acknowledged. Any opinions, findings, and conclusions or recommendations 
expressed in this paper are those of the writers and do not necessarily reflect the views of the funding agency.

\section{References}

[1] J.S. Caygill, F. Davis, S.P.J. Higson, Current trends in explosive detection techniques, Talanta. 88 (2012) 14-29.

[2] R.G. Ewing, D.A. Atkinson, G.A. Eiceman, G.J. Ewing, A critical review of ion mobility spectrometry for the detection of explosives and explosive related compounds, Talanta. 54 (2001) 515-529.

[3] G.A. Eiceman, J.A. Stone, Peer Reviewed: Ion Mobility Spectrometers in National Defense, Anal. Chem. 76 (2004) 390 A-397 A.

[4] A.B. Kanu, C. Wu, H.H. Hill Jr., Rapid pre-separation of interferences for ion mobility spectrometry, Anal. Chim. Acta. 610 (2008) 125-134.

[5] L.M. Matz, P.S. Tornatore, H.H. Hill, Evaluation of suspected interferents for TNT detection by ion mobility spectrometry, Talanta. 54 (2001) 171-179.

[6] T.A. Dickinson, K.L. Michael, J.S. Kauer, D.R. Walt, Convergent, Self-Encoded Bead Sensor Arrays in the Design of an Artificial Nose, Anal. Chem. 71(1999), 2192-2198.

[7] T.M. Swager, The molecular wire approach to sensory signal amplification, Acc. Chem. Res. 31 (1998) 201-207.

[8] A. Kumar, M.K. Pandey, R. Anandakathir, R. Mosurkal, V.S. Parmar, A.C. Watterson, J. Kumar, Sensory response of pegylated and siloxanated 4,8-dimethylcoumarins: A fluorescence quenching study by nitro aromatics, Sensors Actuators B: Chem. 147 (2010) 105-110. 
[9] J. Cho, R. Anandakathir, A. Kumar, J. Kumar, P.U. Kurup, Sensitive and fast recognition of explosives using fluorescent polymer sensors and pattern recognition analysis, Sensors Actuators B: Chem. 160 (2011) 1237-1243.

[10] A.A. Tomchenko, G.P. Harmer, B.T. Marquis, Detection of chemical warfare agents using nanostructured metal oxide sensors, Sensors Actuators B: Chem. 108 (2005) 41-55.

[11] X. Li, J.H. Cho, P. Kurup, Z. Gu, Novel sensor array based on doped tin oxide nanowires for organic vapor detection, Sensors Actuators B: Chem. 162 (2012) 251-258.

[12] J.W. Grate, Acoustic Wave Microsensor Arrays for Vapor Sensing, Chem. Rev. 100 (2000) 2627-2648.

[13] Z. Ying, Y. Jiang, X. Du, G. Xie, J. Yu, H. Wang, PVDF coated quartz crystal microbalance sensor for DMMP vapor detection, Sensors Actuators B: Chem. 125 (2007) $167-172$.

[14] Z. Ying, Y. Jiang, X. Du, G. Xie, J. Yu, H. Tai, Polymer coated sensor array based on quartz crystal microbalance for chemical agent analysis, European Polymer Journal. 44 (2008) $1157-1164$.

[15] J. Huang, Y. Jiang, X. Du, J. Bi, A new siloxane polymer for chemical vapor sensor, Sensors Actuators B: Chem. 146 (2010) 388-394.

[16] E. Pasquinet, C. Bouvier, F. Thery-Merland, L. Hairault, B. Lebret, C. Méthivier, C.M. Pradier, Synthesis and adsorption on gold surfaces of a functionalized thiol: elaboration and test of a new nitroaromatic gas sensor, J. Colloid Interface Sci. 272 (2004) 21-27.

[17] J.W. Grate, Hydrogen-Bond Acidic Polymers for Chemical Vapor Sensing, Chem. Rev. 108 (2008) 726-745. 
[18] W. He, Z. Liu, X. Du, Y. Jiang, D. Xiao, Analytical application of poly\{methyl[3-(2hydroxy-3,4-difluoro)phenyl]propyl siloxane $\}$ as a QCM coating for DMMP detection, Talanta 76 (2008) 698-702 .

[19] J. Huang, Y. Jiang, X. Dua, J. Bi, A new siloxane polymer for chemical vapor sensor, Sensors and Actuators, B: Chemical 146 (2010) 388-394.

[20] E.J. Houser, T.E. Mlsna, V.K. Nguyen, R. Chung, R.L. Mowery, R. Andrew McGill, Rational materials design of sorbent coatings for explosives: applications with chemical sensors, Talanta. 54 (2001) 469-485.

[21] T.W. Campbell, W.A. McAllister, M.T. Roger, The Electric Moments of Some Derivatives of Azobenzene, J. Am. Chem. Soc. 75 (1953) 864-865.

[22] M.A. Rodriguez, S.E. Braslavsky, Photoisomerization of Azobenzenecarboxylic Acids and Their Potassium Salts: Evidence of Structural Volume Changes Associated with Hydrogen Bond Formation, J. Phys. Chem. A. 103 (1999) 6295-6300.

[23] T. Ponrathnam, J. Cho, P. Kurup, R. Nagarajan, J. Kumar, Investigation of QCM Sensors with Azobenzene Functionalized Coatings for the Detection of Nitroaromatics, - Journal of Macromolecular Science, Part A. 48 (2011), 1031-1037.

[24] D. Poullain, E. Pasquinet, and L. Hairault, inventors; Commissariat A L'energie Atomique, assignee. Chemical sensors comprising aniline polysiloxanes as sensitive materials and use thereof for detecting of assaying nitro compounds. US Patent 7,968,343. 2011 June 28. Washington, DC.

[25] S. Liu, T. Ponrathnam, H. Sun, R. Nagarajan, J. Kumar, Z. Gu, P. Kurup, Detection of Explosive Vapors by Surface Acoustic Wave Sensors Containing Novel Siloxane Based Coatings, Journal of Macromolecular Science, Part A. 47 (2010) 1172-1175. 
[26] I.H. Witten, E. Frank, Data Mining: Practical Machine Learning Tools and Techniques, Morgan Kaufmann, 2005.

[27] G. Sauerbrey, Verwendung von Schwingquarzen zur Wägung dünner Schichten und zur Mikrowägung, Text. 155 (1959) 206-222.

[28] S. Clavaguera, P. Montméat, F. Parret, E. Pasquinet, J. Lère-Porte, L. Hairault, Comparison of fluorescence and QCM technologies: Example of explosives detection with a $\pi$-conjugated thin film, Talanta. 82 (2010) 1397-1402.

[29] M. Roth, Solubility parameter of poly(dimethyl siloxane) as a function of temperature and chain length, Journal of Polymer Science Part B: Polymer Physics. 28 (1990) 2715-2719. 


\section{TABLE CAPTIONS}

Table 1: Change in frequency on coating the polymers onto the QCM crystals

Table 2: Chemical Properties of the Analytes tested

Table 3: Comparison of the sensor responses to various analytes

Table 4: Effect of dipole moment on the normalized sensor response

Table 5: Prediction of analyte using CART based decision tree 
Table 1.

\begin{tabular}{cc}
\hline Coatings & $\begin{array}{c}\text { Change in Frequency on addition } \\
\text { of polymer coatings (Hz) }\end{array}$ \\
\hline PDMS-AN & 1360 \\
\hline PDMS-AN-NT & 1210 \\
\hline PDMS-AN-CA & 1590 \\
\hline
\end{tabular}


Table 2.

Hildebrand

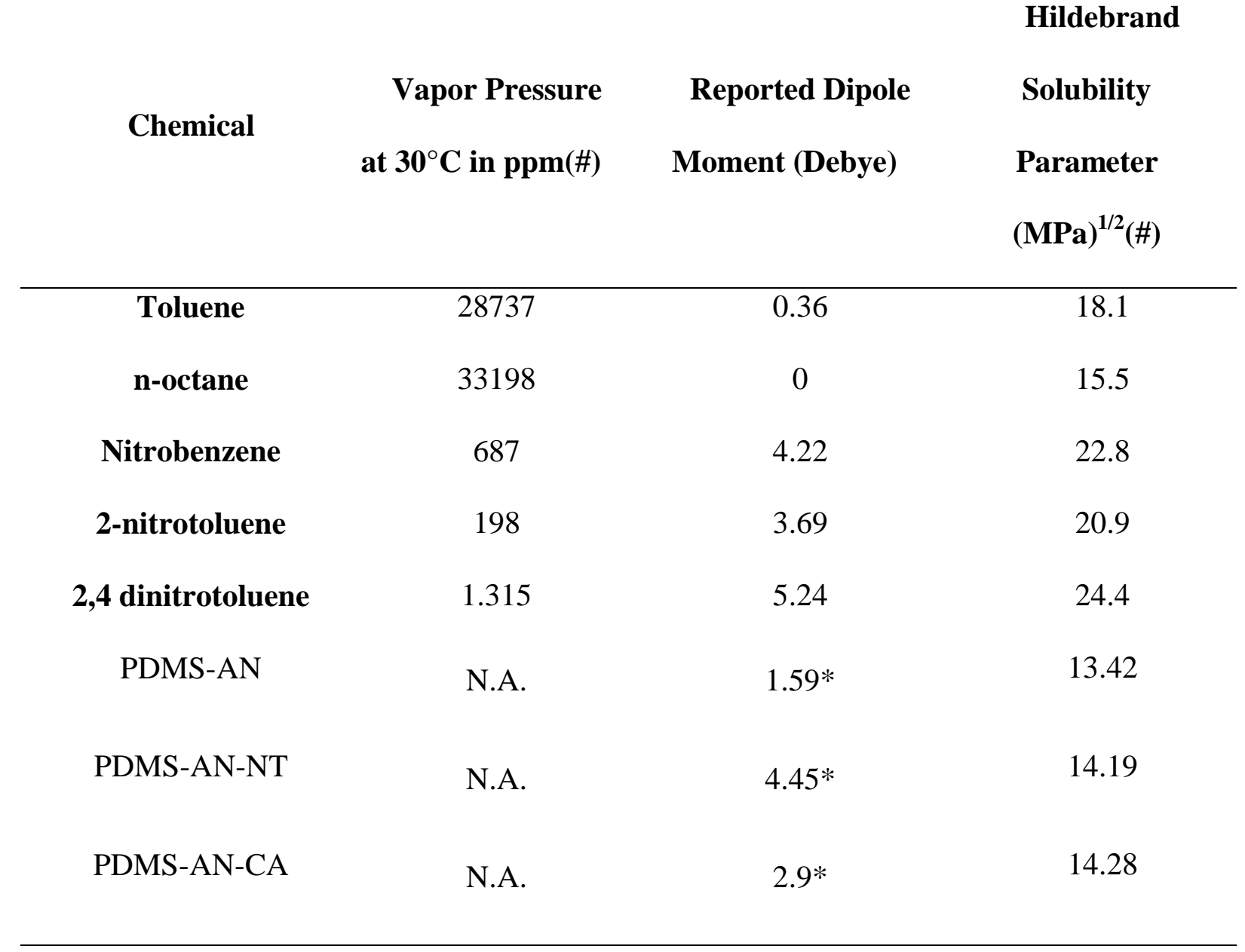

*Dipole moment of the aromatic group

\# Estimated using HSPIP softwware 
Table 3.

\begin{tabular}{|c|c|c|c|c|c|c|}
\hline \multirow[t]{2}{*}{ Analyte } & \multicolumn{3}{|c|}{$\begin{array}{c}\text { Maximum frequency change } \\
(\mathrm{Hz})\end{array}$} & \multicolumn{3}{|c|}{$\begin{array}{c}\text { Max. Frequency Change } \\
\text { Normalized to Vapor Pressure } \\
\text { (Hz/ppm) }\end{array}$} \\
\hline & $\begin{array}{l}\text { PDMS- } \\
\text { AN }\end{array}$ & $\begin{array}{l}\text { PDMS- } \\
\text { AN-NT }\end{array}$ & $\begin{array}{l}\text { PDMS- } \\
\text { AN-CA }\end{array}$ & $\begin{array}{l}\text { PDMS- } \\
\text { AN }\end{array}$ & $\begin{array}{l}\text { PDMS- } \\
\text { AN-NT }\end{array}$ & $\begin{array}{l}\text { PDMS- } \\
\text { AN- CA }\end{array}$ \\
\hline Toluene & 660 & 930 & 912 & 0.022 & 0.032 & 0.031 \\
\hline n-Octane & 2100 & 1660 & 1000 & 0.063 & 0.050 & 0.030 \\
\hline Nitrobenzene & 1018 & 2010 & 1263 & 1.481 & 2.925 & 1.838 \\
\hline $2-$ & 1272 & 2211 & 1390 & & 11.167 & 7.020 \\
\hline Nitrotoluene & & & & 6.424 & & \\
\hline 2,4 DNT & 40 & 52 & 43 & 30.418 & 39.543 & 32.699 \\
\hline
\end{tabular}


Table 4.

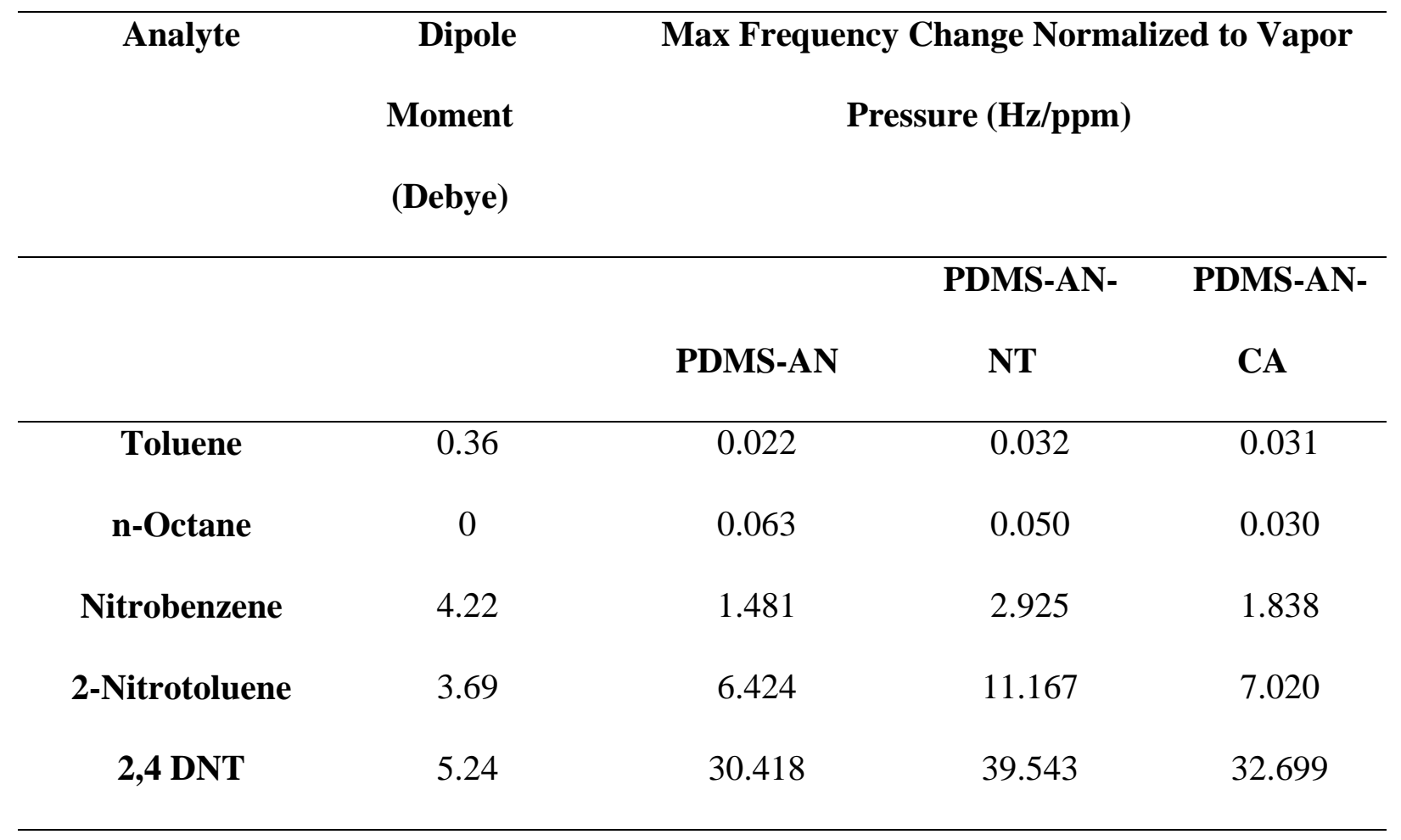


Table 5.

Actual

\begin{tabular}{|c|c|c|c|c|c|}
\hline Predicted & Toluene & n-Octane & NB & 2-NT & 2,4 DNT \\
\hline Toluene & 5 & & & & \\
\hline n-Octane & & 5 & & & \\
\hline NB & & & 5 & 1 & \\
\hline 2-NT & & & & 4 & \\
\hline 2,4 DNT & & & & & 5 \\
\hline
\end{tabular}

2015-03-13

Revised Draft 


\section{Biographies}

Timothy Ponrathnam is currently a doctoral student in the Department of Plastics Engineering at the University of Massachusetts Lowell (UML). He completed his undergraduate engineering degree (B.E.) from the University of Pune, India in 2006 studying Polymer Engineering and a Masters in Chemistry at UML in 2010. Prior to joining UML for graduate studies, he worked as a project researcher at the National Chemical Laboratory (NCL) India. His research interests include the development of novel polymer coatings that are utilized to enhance the sensitivity of sensors towards the analytes of interest such as nitroaromatic explosive vapors.

Junghwan Cho is currently a postdoctoral researcher at the University of Massachusetts Lowell, U.S.A. He received a B.S. in Instrumentation and Control Engineering from Gyeongsang National University, South Korea in 2001, then received an M.E. and a Ph.D. in Electronic Engineering from Kyungpook National University, Daegu, South Korea in 2003 and 2008, respectively. His research interests include pattern recognition techniques, fuzzy systems, and artificial neural networks applied to electronic nose and tongue devices.

Pradeep U. Kurup is a Professor in the Department of Civil and Environmental Engineering at the University of Massachusetts Lowell. He received his B.Tech. Civil Engineering in 1985 from the University of Kerala and obtained his M.Tech. from the Indian Institute of Technology Madras (1987). He holds a Ph.D. in Civil and Environmental Engineering (1993) from Louisiana State University (LSU). Dr. Kurup has conducted extensive research in geotechnical \& environmental site characterization; sensor integration; pattern recognition using intelligent models; multi-sensor data fusion; and artificial olfaction. Dr. Kurup is a member of several professional societies, and is a registered Professional Engineer in the State of Louisiana.

Jayant Kumar is a Professor of Physics at University of Massachusetts Lowell. He did his PhD in physics at Rutgers University, USA. He also serves as the director of the Center for Advanced

2015-03-13

Revised Draft 
Materials (CAM) at the University of Massachusetts Lowell. His research interests include optical sensors, nonlinear optics, organic solar cells, etc.

Ramaswamy Nagarajan is an associate professor in the Department of Plastics Engineering at University of Massachusetts Lowell. He received his Ph.D. in Polymer Science from the University of Massachusetts Lowell. His research interests include developing 'Greener' routes to advanced functional materials (electronic, photo-responsive polymers) and therapeutic materials, biocatalytic polymerization, polymers from renewable resources, mechanistic aspects of enzyme catalysis, developing novel eco-friendly biomimetic catalysts, RF-based sensors for wireless structural health monitoring and sensors for detecting explosives in air and toxic metals in soil and water. He also serves as the co-director of HEROES which is a joint research and development initiative of UMass Lowell and the U.S. Army Natick Soldier Research Development and Engineering Center (NSRDEC). 


\section{FIGURE CAPTIONS}

Fig 1. Structure and dipole moments of Nitroaromatic analytes evaluated in this study

Fig 2. Structure of p-nitroazobenzene functionalized poly(propylene glycol) coating

Fig 3. Structure of the precursor (unfunctionalized) poly(propylene glycol) coating

Fig. 4. Synthesis of the precursor polymer PDMS-AN

Fig. 5. Synthetic scheme describing preparation of the PDMS-AN-NT polymer

Fig. 6. Synthetic scheme describing preparation of the PDMS-AN-CA polymer

Fig. 7. Schematic of the test setup

Fig. 8. Response of the polymer coated QCM sensor to 2-Nitrotoluene

Fig. 9. Response of the coated QCM sensors to toluene vapor

Fig. 10. Response of the coated QCM sensors to octane vapor

Fig. 11. Response of the coated QCM sensors to nitrobenzene vapor

Fig. 12. Response of the coated QCM sensors to 2-nitrotoluene vapor

Fig. 13. Response of the coated QCM sensors to 2,4 DNT vapor

Fig. 14. Comparison of the Sensor Response to various analytes tested

Fig. 15. Principle Component Analysis of the sensor array response to various analytes

Fig. 16. Classification of based on CART analysis 


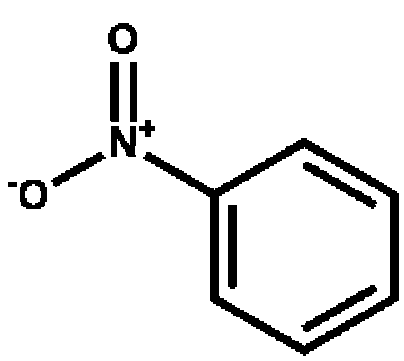

nitrobenzene

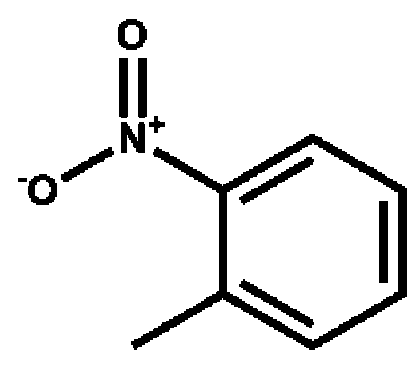

2-nitrotoluene

Dipole Moment 3.69 Debye

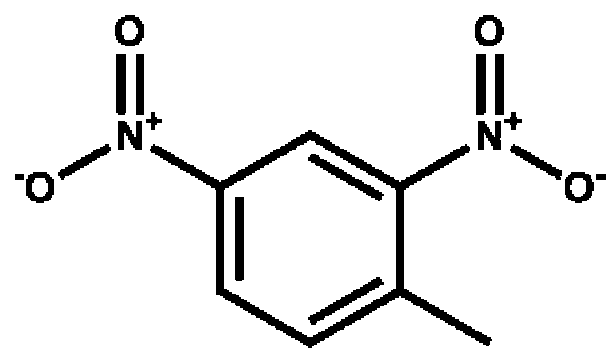

2,4 dinitrotoluene

Dipole Moment 5.24 Debye

Fig.1: 


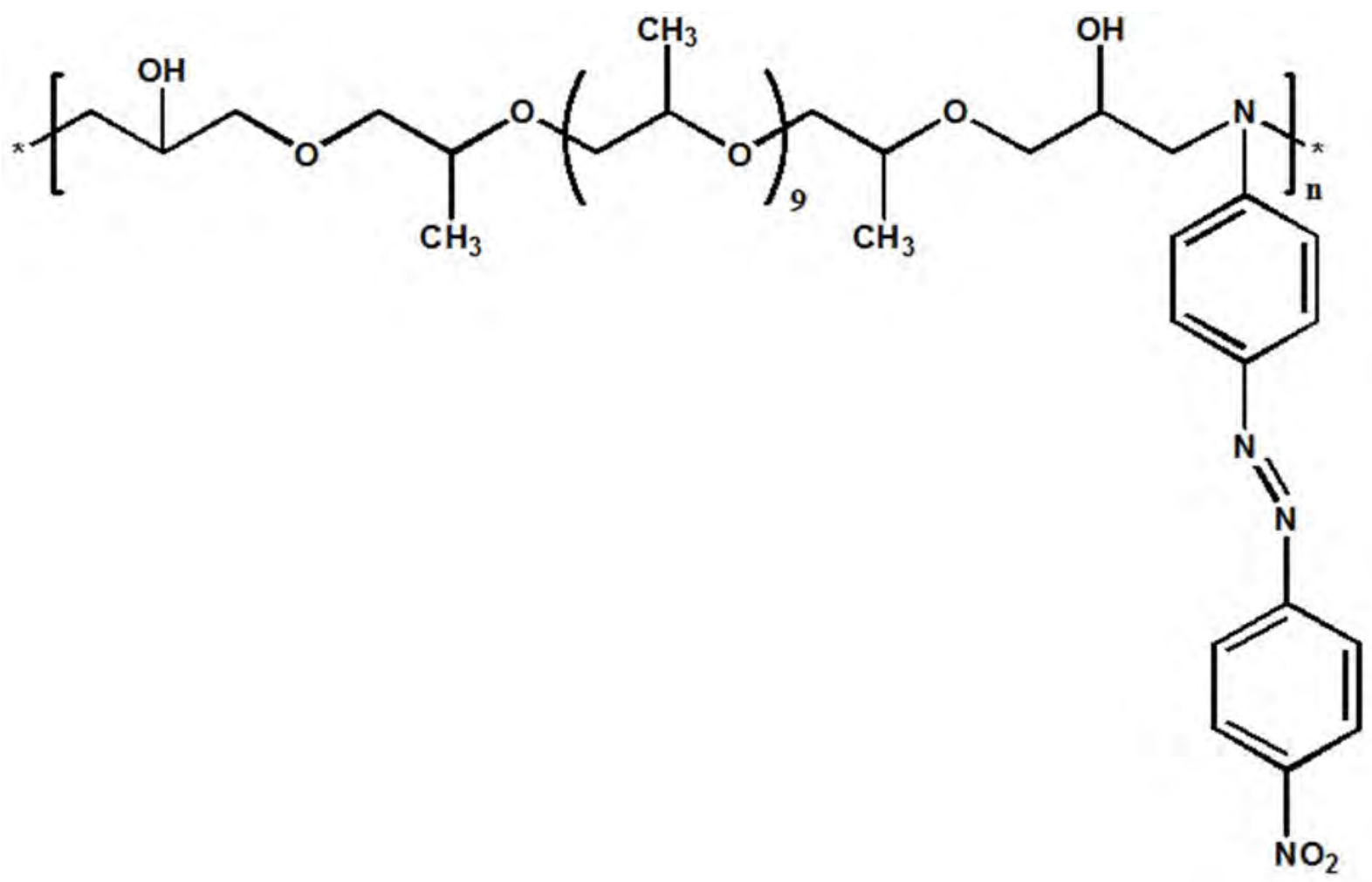

Fig.2: 


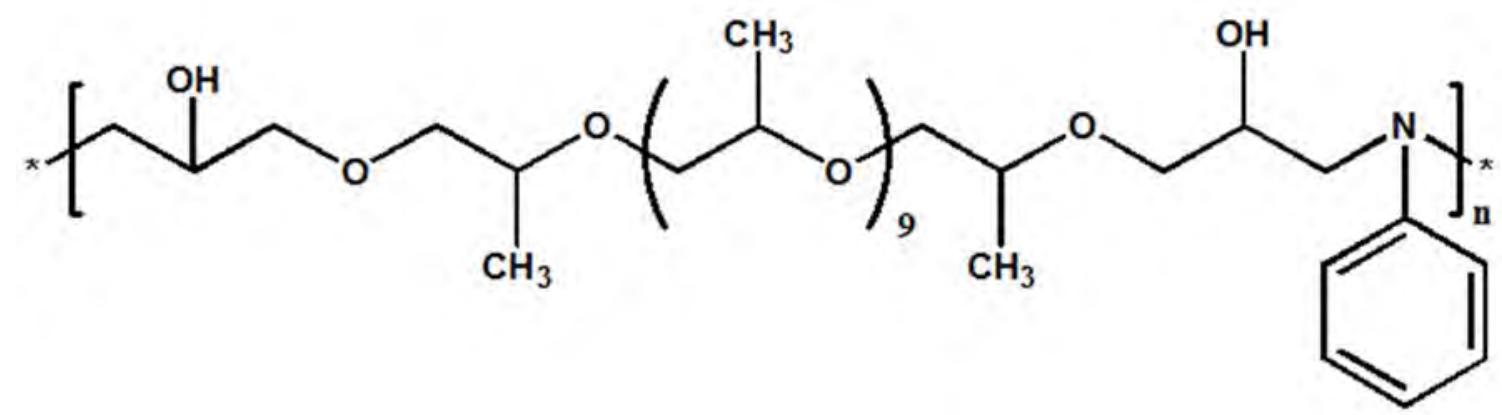

Fig. 3: 
<smiles>C[Si](C)(CCCOCC1CO1)O[Si](C)(C)O[Si](C)(C)CCCOCC1CO1</smiles><smiles>CC(Cl)(Br)C([Ge])c1ccccc1N</smiles><smiles>CC(C)(I)C(O)COCCC[Si](C)(C)O[Si](C)(C)O[Si](C)(C)CCCOCCC(O)N1CCC1(C)C</smiles>

Fig.4: 

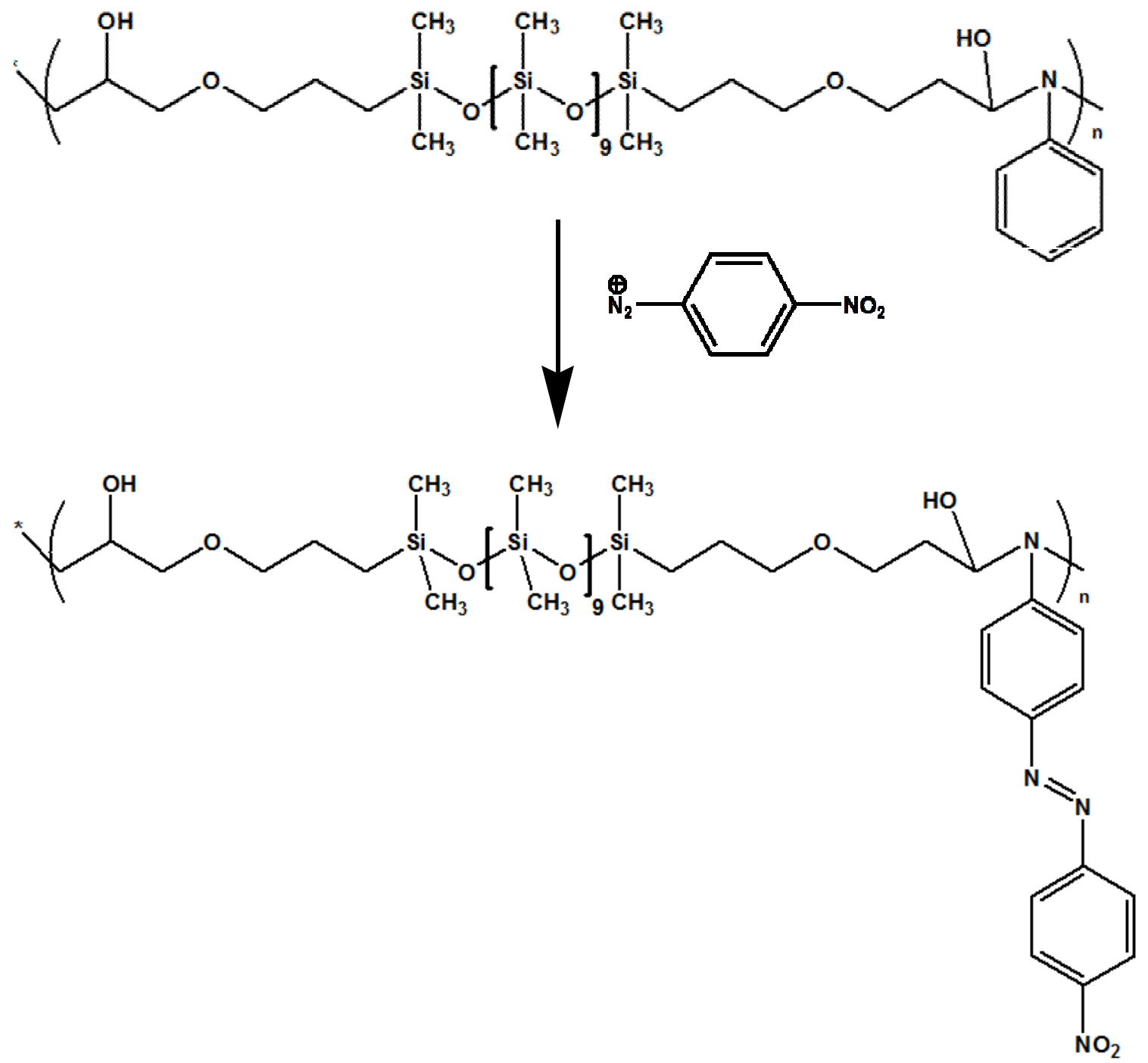

Fig. 5: 


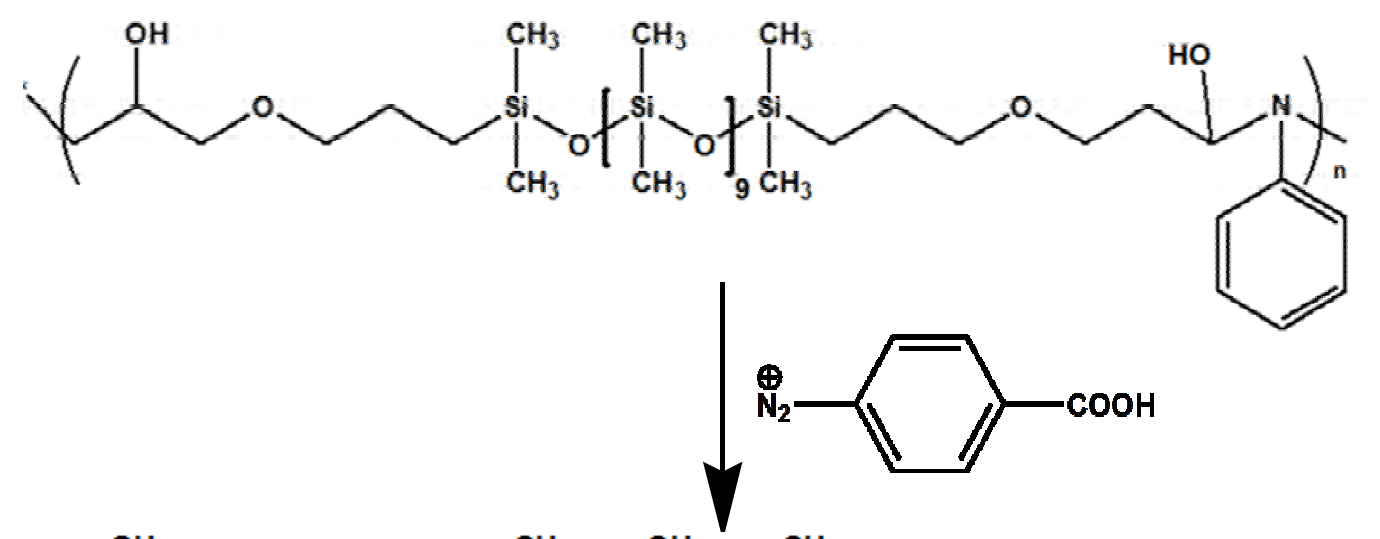<smiles>CCOCCC[Si](C)(C)O[Si](C)(C)O[Si](C)(C)CCCOCC(O)C(C)(C)CC</smiles><smiles>CCC(O)N(C)c1ccc(/N=N/c2ccc(C(=O)O)cc2)cc1</smiles>

Fig. 6: 


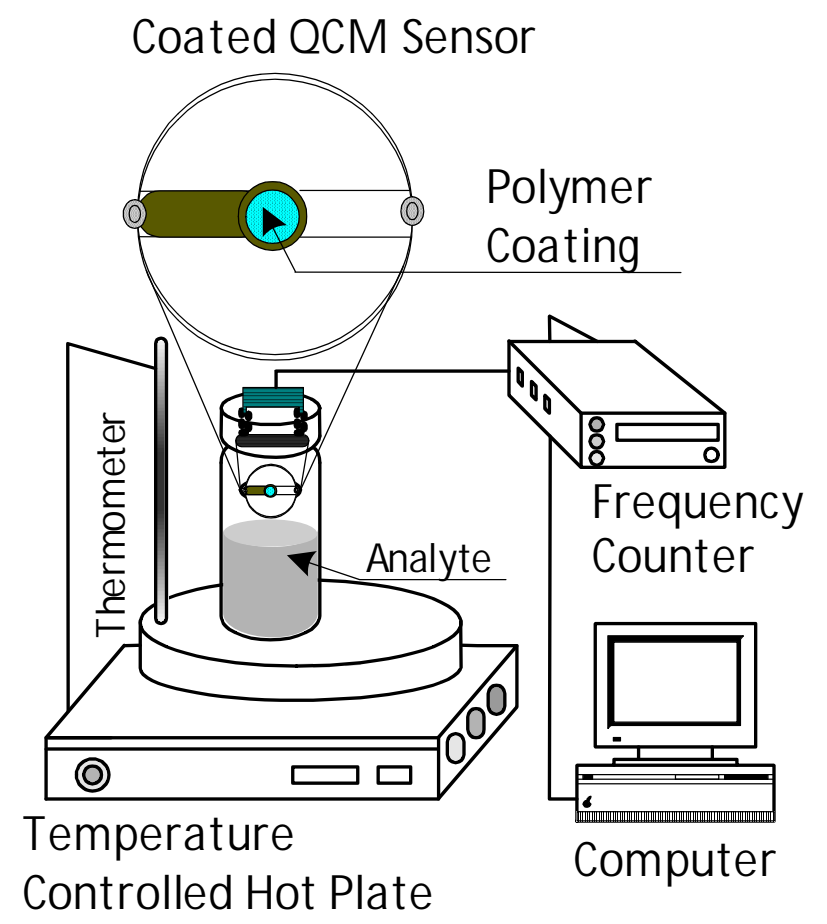

Fig. 7: 


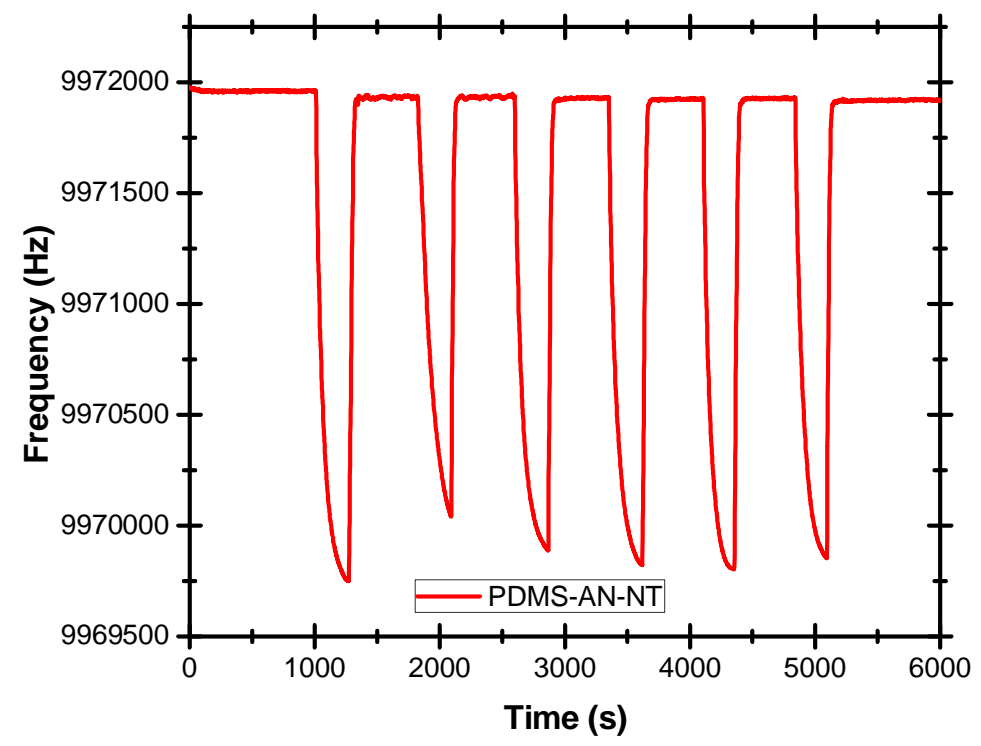

Fig. 8: 


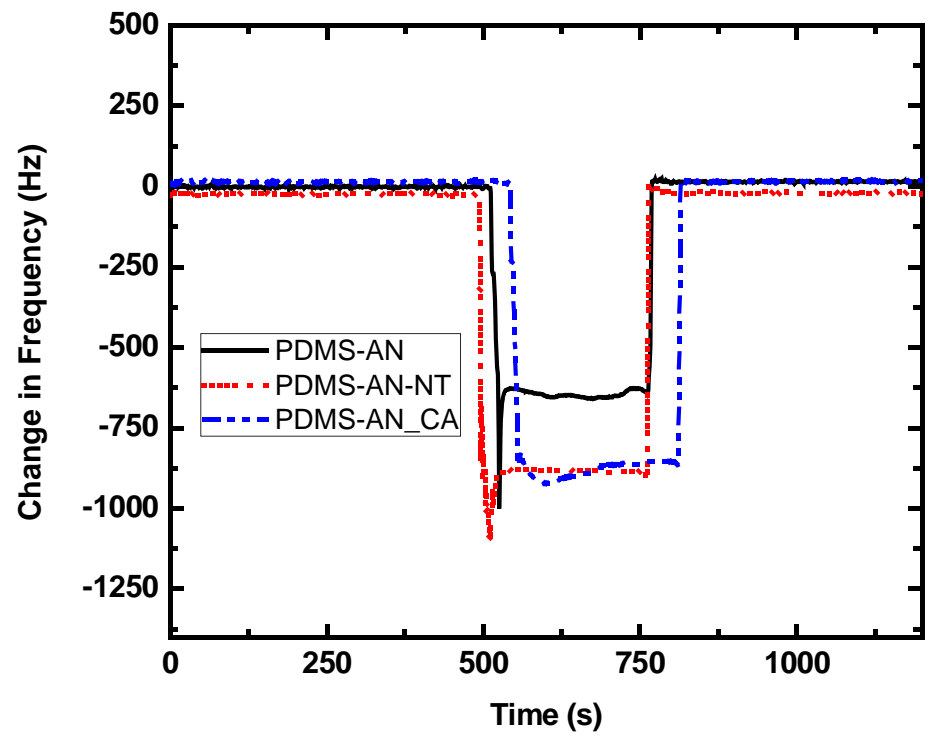

Fig. 9: 


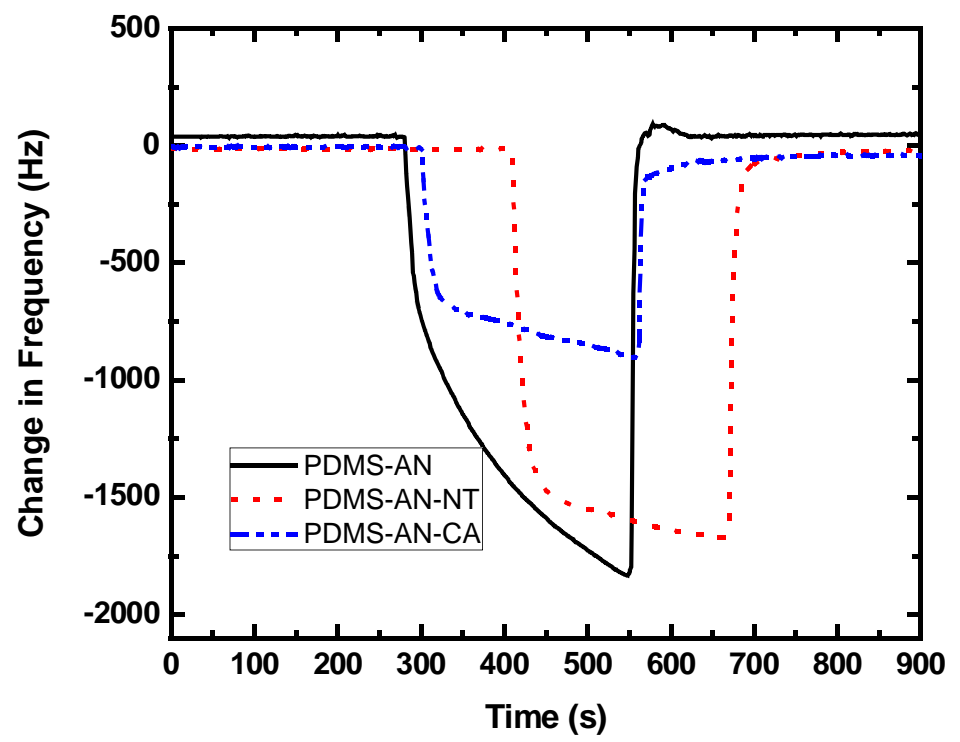

Fig. 10: 


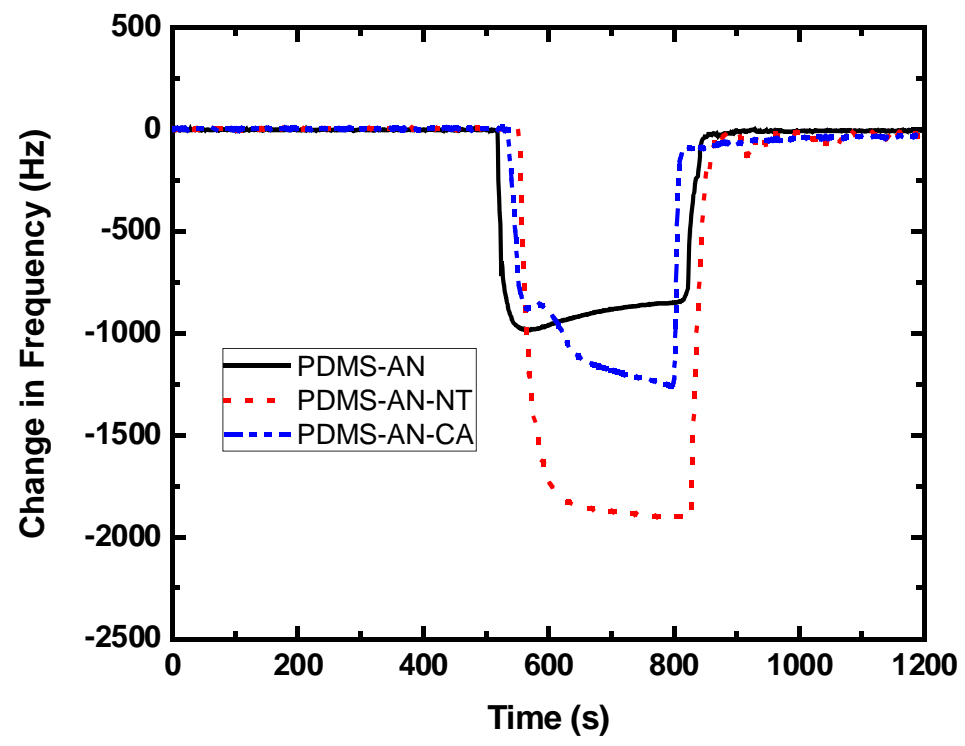

Fig. 11: 


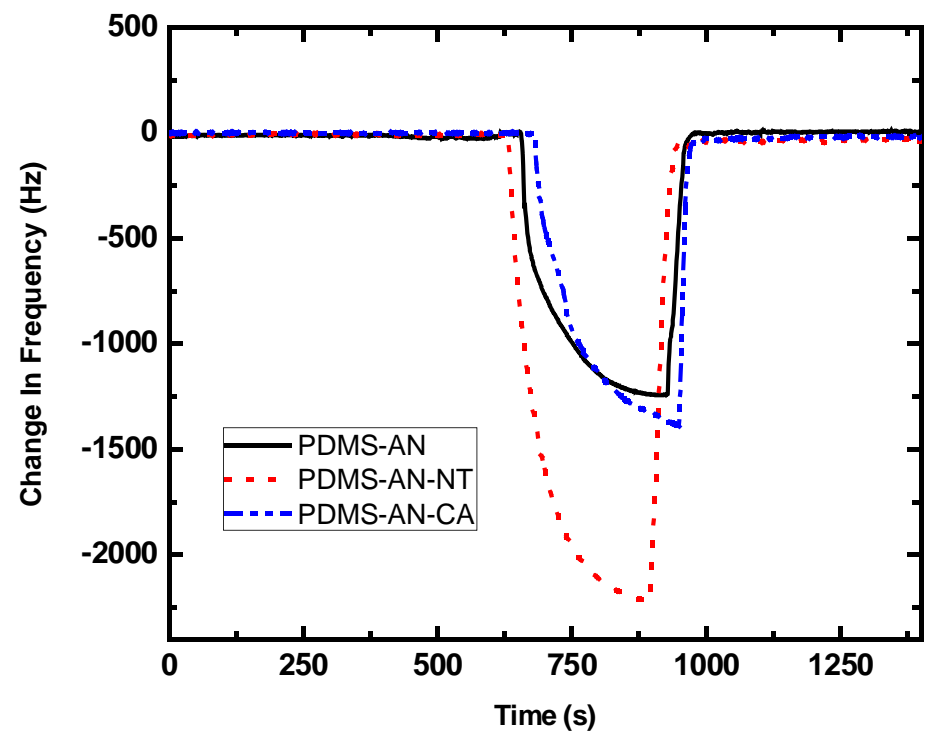

Fig. 12: 


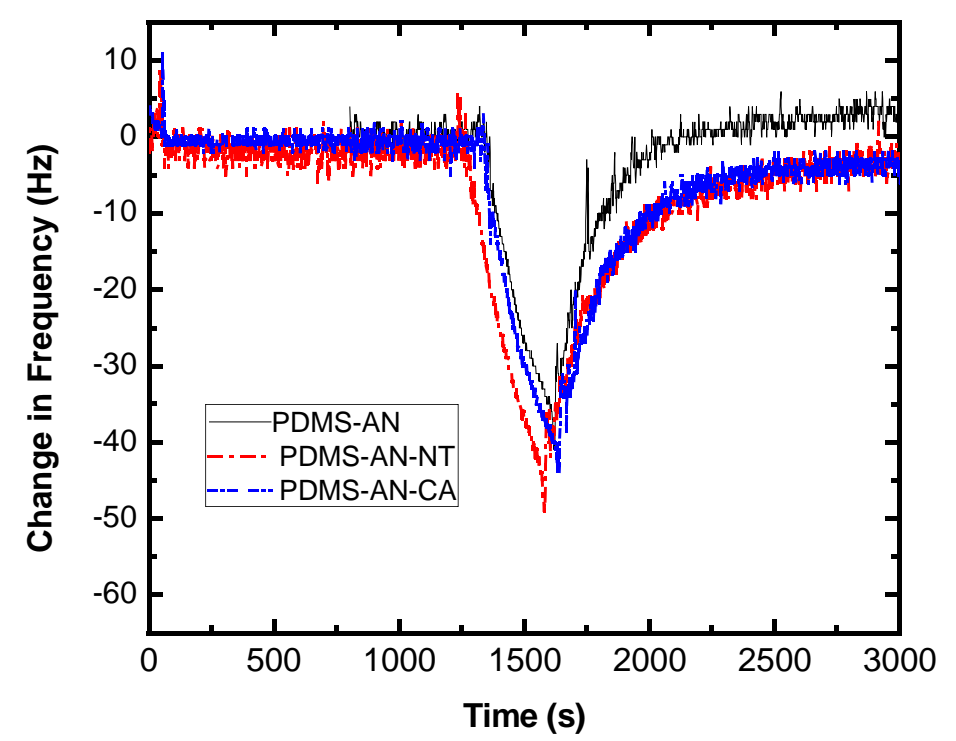

Fig. 13: 


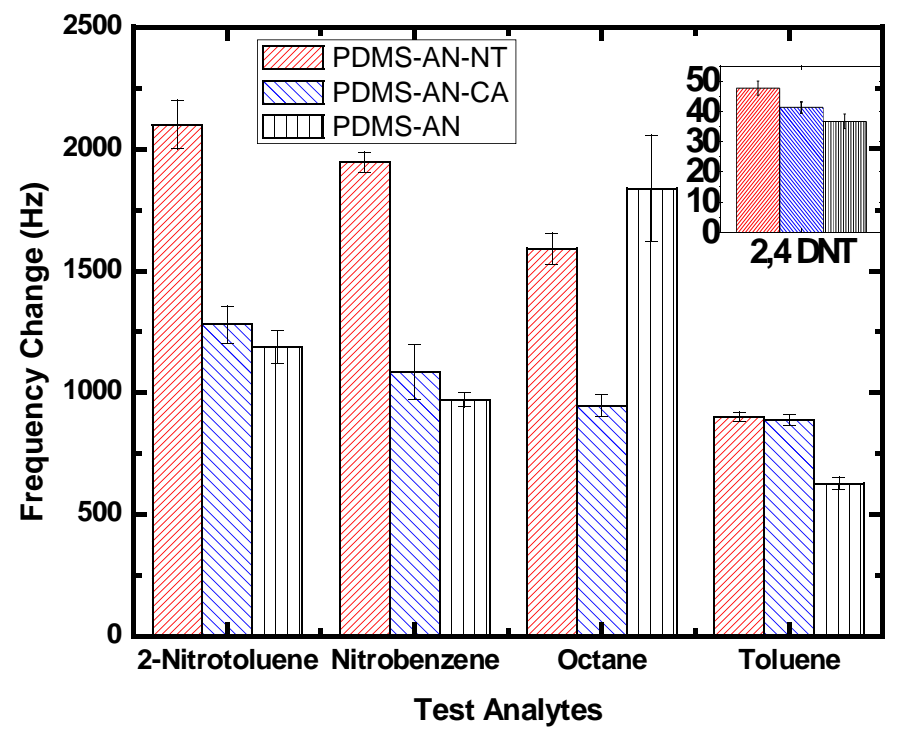

Fig. 14: 


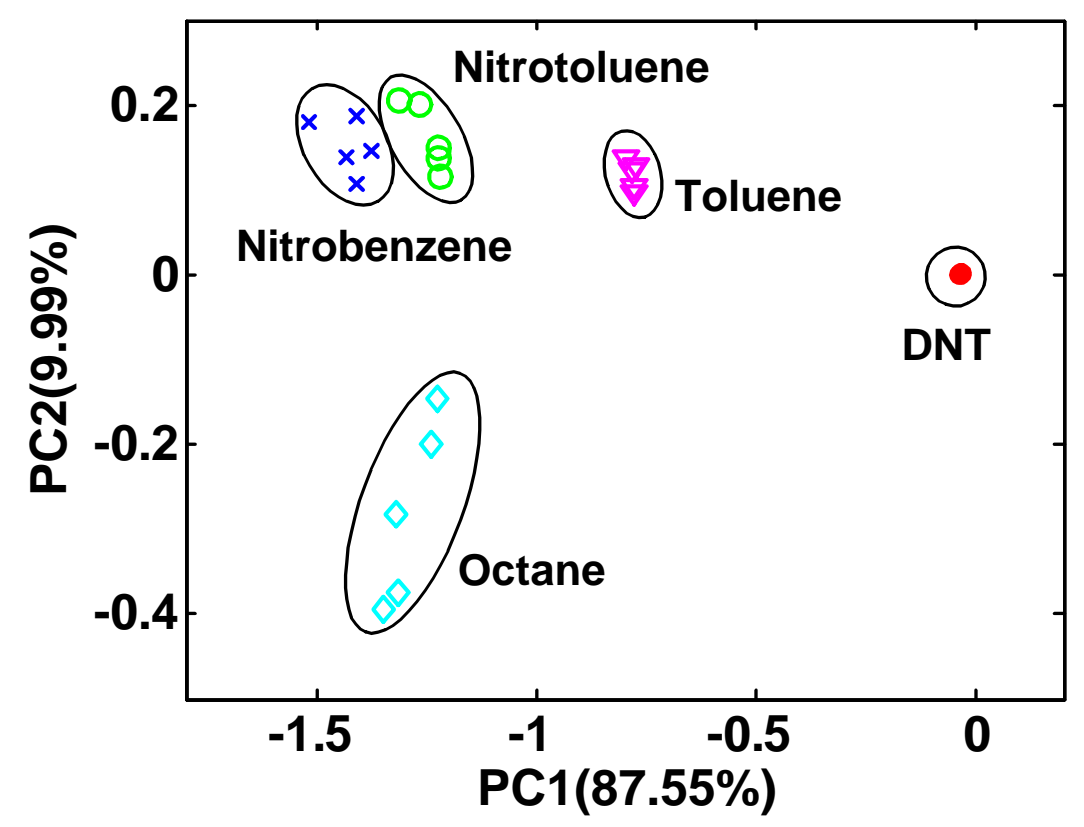

Fig. 15: 


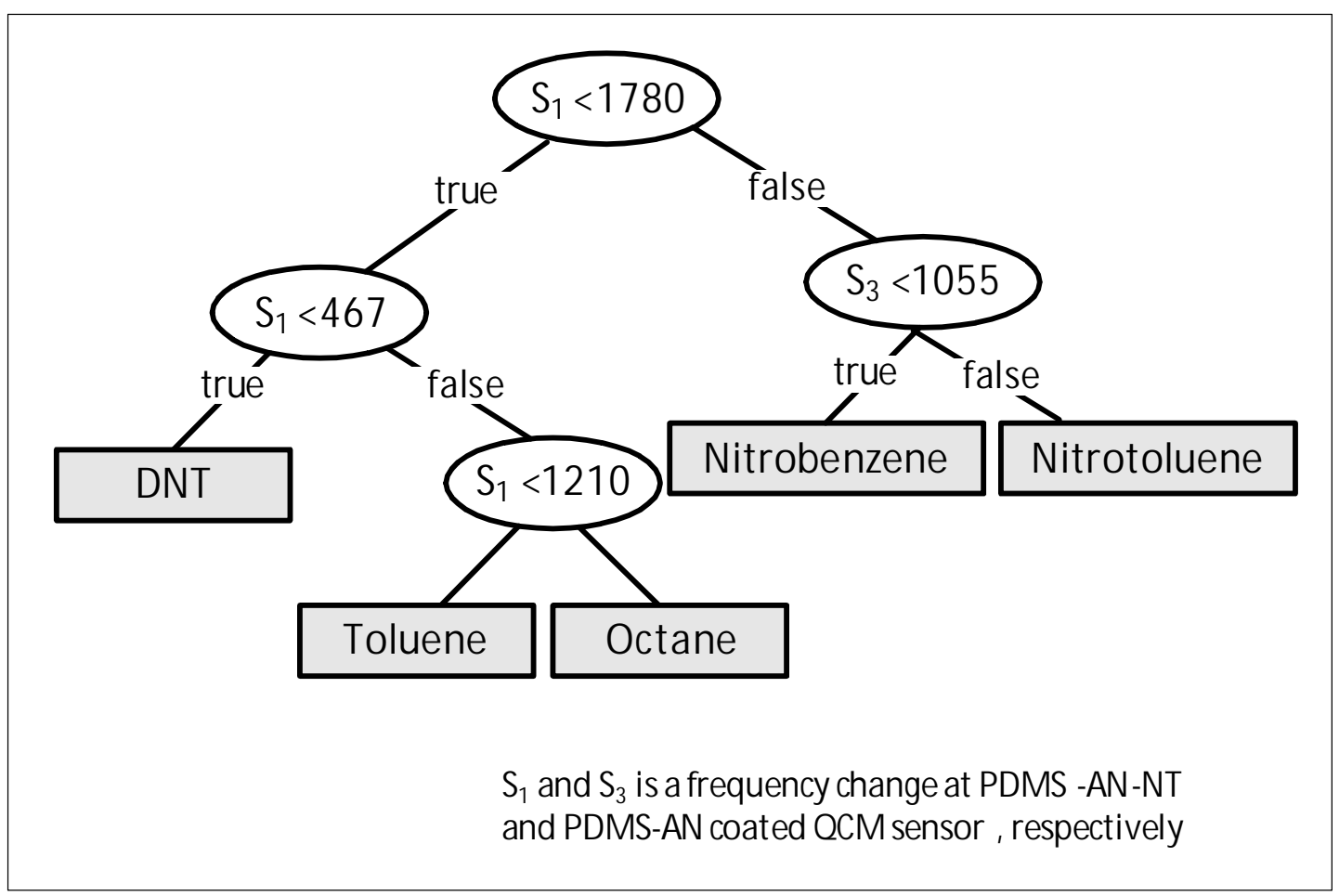

Fig. 16: 